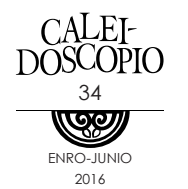

\title{
Búsqueda de sensaciones y consumo de drogas en adolescentes mexicanos
}

CITLALI PÉREZ DE LA BARRERA'

\section{RESUMEN}

El objetivo de este estudio fue identificar si existían diferencias en el nivel de búsqueda de sensaciones en cuanto al consumo de drogas en adolescentes escolarizados. La muestra estuvo conformada por 386 estudiantes de nivel medio superior en el estado de Morelos, México. Para medir la búsqueda de sensaciones, se utilizó una escala validada en población mexicana conformada por tres factores: 1) gusto por el riesgo, con 8 reactivos y un alfa de 0.88 ; 2) emociones fuertes, con 4 reactivos y una alfa de 0.70 ; y 3) cautela, con 4 reactivos y un alfa de 0.65 . Los resultados mostraron diferencias significativas en la frecuencia de consumo de alcohol en el último año y mes entre los estudiantes consumidores y no consumidores, donde estos últimos presentan un menor nivel de búsqueda de sensaciones. Respecto a las drogas ilegales, los estudiantes que nunca las han consumido obtuvieron un puntaje más bajo en su nivel de búsqueda de sensaciones, en comparación con los estudiantes con consumo de alguna droga ilegal alguna vez en su vida. Además, se identificaron diferencias estadísticamente significativas en los estudiantes que han consumido marihuana e inhalables alguna vez, en comparación con los que nunca han consumido. Estos hallazgos sugieren la necesidad de desarrollar programas que inclu-

1 Doctora en Psicología por la Facultad de Psicología. Universidad Nacional Autónoma de México.salutmc@yahoo.com.
C
A L $\quad E$
D
S
$C O P$ I
O
- 13 
yan el fortalecimiento de la habilidad de autocontrol por ser un factor protector del consumo de drogas en adolescentes.

Palabras clave: búsqueda de sensaciones, drogas, adolescentes, autocontrol, prevención.

\section{ABSTRACT}

The objective of this study was to identify if there were differences in the sensation seeking level towards drug consumption among adolescents. The sample was made up of 386 students from the State of Morelos, Mexico. To measure sensation seeking a scale validated among Mexican population was used conformed by three factors: 1) liking for risk, with 8 items and an alpha of $0.88 ; 2$ ) strong emotions, with 4 items and an alpha of 0.70 ; y 3) caution, with 4 items and an alpha of 0.65 . Results showed significant differences in the alcohol consumption frequency during the last year and last month among consumers and non consumers' students, where these last ones got a lower score in their sensation seeking level. Referring to illegal drugs, the students that had never consumed illegal drugs got a lower score in their sensation seeking level compared to those who had consumed them; in addition, significant differences were found among students that had consumed cannabis and inhalants once in their lives compared to those who had never done. These findings suggest the need to develop programs that include the enhancing of self-control skill as a protective factor from drug abuse among adolescents.

Key words: Sensation seeking, drugs, adolescents, self-control, prevention.

La adolescencia ha sido definida como un periodo entre la niñez y la adultez durante la cual los jóvenes experimentan un rápido crecimiento físico y cognitivo. Asimismo, este periodo se caracteriza especialmente por la experimentación de situaciones novedosas, así como por un aumento en las conductas de riesgo como el consumo de drogas, la conducción temeraria, la práctica de deportes extremos y las relaciones sexuales sin protección (Dahl, 2008; Gervilla y Palmer, 2009; Jackson et al., 2012; Palacios y Cañas, 2010; Pérez de la Barrera, en prensa; Sales et al., 2013; Terzian, Andrews y Moore, 2011; Zuckerman, 2007).

Respecto a la morbilidad en México, la última Encuesta Nacional de Adicciones (ENA, 2011) reportó las últimas tendencias en cuanto al 
abuso de drogas: la edad promedio de inicio en el consumo de tabaco es de 14.1 años. En el grupo de edad de 12-17 años se identificó que $16.4 \%$ de hombres y $7.1 \%$ de mujeres tienen un consumo activo, y un promedio de 4 cigarrillos diarios, y 7.1\% tienen niveles altos de adicción. Respecto al consumo de alcohol en el último año, 17.3\% de hombres y $11.7 \%$ de mujeres tienen un consumo alto, y $6.2 \%$ de los hombres y $2 \%$ de las mujeres tienen dependencia. En cuanto al uso de drogas ilegales, se identifican la marihuana (1.3\%) y la cocaína $(0.4 \%)$ como las preferidas por esta población, seguidas por los inhalables (0.3\%).

La investigación sobre el estudio de factores de riesgo relacionados con el consumo de drogas en adolescentes ha puesto de relieve características de personalidad, donde la más ampliamente estudiada ha sido la búsqueda de sensaciones (Fuhrel y Kalichman, 2006). La búsqueda de sensaciones (BS) ha sido definida como una "disposición psicobiológica caracterizada por la necesidad de experiencias variadas, novedosas e intensas y una tendencia a involucrarse en situaciones de riesgo para lograr tales experiencias" (Zuckerman, 1994: 27). Zuckerman y Neeb $(1980)$ y Zuckerman $(1984 ; 1994)$ propusieron un modelo psicobiológico que incluye un conjunto de monoaminas, enzimas y metabolitos que, interactuando de modo complejo, podrían ser el sustrato biológico de la BS. La BS está asociada con una reactividad dopaminérgica fuerte y con actividades serotoninérgicas y noradrenérgicas débiles (Zuckerman, 2007). Durante la adolescencia, el aumento de dopamina en los centros de recompensa subcorticales favorece la atracción por la novedad y la emoción por las experiencias inmediatas. El sistema dopaminérgico, que es eminentemente sensible para detectar las recompensas novedosas, motiva la búsqueda de esas experiencias (Zuckerman, 1994).

La BS relacionada con el consumo de drogas ha recibido una considerable atención por parte de la comunidad científica con estudios que aportan evidencia de una mayor BS en los adolescentes que abusan de las drogas (Belin et al., 2008; Desrichard y Denarié, 2005; Pokhrel et al., 2010; Schmidt et al., 2004).

Por otra parte, la evidencia neurocientífica ha documentado que la mayoría de las conductas de riesgo son palpables, en principio, en la pubertad -10 a 12 años-, cuando los cambios neuroendocrinos están ocurriendo, especialmente en los hombres (Martin et al., 2002; Steinberg, 2008). Asimismo, los niveles elevados de testosterona se han asociado con la toma de riesgo en hombres; en mujeres, están 
vinculados con la tendencia a afiliarse con pares con conductas desviadas. Además, se ha identificado que la testosterona y los estrógenos tienen un efecto energizante, el cual favorece la experimentación con drogas y las prácticas sexuales (National Research Council [NCR], 2011). A este respecto, cabe resaltar que la corteza prefrontal es el sitio de control de las funciones ejecutivas que empiezan a emerger temprano en la vida y continúan desarrollándose en la adultez. Estos centros ejecutivos son críticos, ya que permiten al individuo ejercer control sobre un amplio rango de respuesta: modulan la sensibilidad hacia diferentes tipos de recompensa, identifican la importancia del estímulo y ejercen control sobre los impulsos y las respuestas sociales y emocionales. Sin embargo, el desarrollo de la corteza prefrontal es gradual y no se completa hasta la adultez; en contraparte, el sistema límbico -el centro de recompensa del cerebro- presenta un desarrollo pronunciado en la adolescencia.

Esta disparidad entre el desarrollo gradual de la corteza prefrontal y el desarrollo más rápido del sistema límbico que gobierna la BS, ayuda a explicar por qué los adolescentes son más proclives a tomar riesgos (Casey, Getz y Galván, 2008; NCR, 2011). Otros estudios han mostrado diferencias en las respuestas de los cerebros de adultos y adolescentes hacia los estímulos, así como percepciones de riesgo y recompensa. Los adolescentes parecen más influidos por situaciones cargadas de estrés, diversión o emoción cuando toman decisiones, y como resultado pueden encontrar el consumo de drogas más gratificante que los adultos, percibiendo, además, un mejor desempeño social cuando están bajo los efectos del alcohol. También son más sensibles a los efectos adversos de estas sustancias (NRC, 2011). Además, los adolescentes difieren de los adultos en su capacidad para controlar sus impulsos cuando se encuentran en situaciones emocionales intensas, por ello, pueden estar conscientes de que una decisión no es prudente, pero se sienten incapaces de resistir el impulso; en cambio, cuando los adultos toman malas decisiones es porque han identificado las razones que apoyan esa decisión (Casey et al., 2008).

Debido al considerable aumento del consumo de drogas en México en población adolescente, y la abundante evidencia que relaciona la BS con este consumo, el objetivo de este estudio fue explorar la relación entre estas dos variables e identificar si existen diferencias en el nivel de BS por consumo de drogas en adolescentes estudiantes mexicanos. 
MÉTODO

Participantes

Se trabajó con 168 (43.5\%) hombres y 218 (56.5\%) mujeres adolescentes del Estado de Morelos, México, estudiantes de bachillerato en una institución privada, donde $34.4 \%$ eran de cuarto semestre, $33.6 \%$ de quinto semestre y $32 \%$ de sexto semestre. Los estudiantes fueron seleccionados de manera intencional y su rango de edad osciló entre los 14 y 20 años, con una media de edad de 16.17 y una desviación estándar de 2.4. Todos los estudiantes eran solteros.

\section{Instrumento}

Para medir la BS se utilizó el instrumento validado por Contreras (2006) en población mexicana. Este instrumento se encuentra conformado por tres factores: 1) gusto por el riesgo: se refiere a la búsqueda de situaciones en las que el individuo se pone en riesgo; con un alfa de 0.88 con 8 reactivos (ejemplo: "hago cosas arriesgadas sólo porque son emocionantes"); 2) emociones fuertes: se refiere a la falta de medición de las consecuencias en la BS de riesgo; con un alfa de 0.70 con 4 reactivos (ejemplo: "hago lo que me gusta sin pensar en las consecuencias"); y 3) cautela: se refiere a la medición de las consecuencias y a no buscar situaciones riesgosas, con un alfa de 0.65 con 4 reactivos (ejemplo: "pienso las cosas antes de hacerlas"). Los tres factores explican $50.45 \%$ de la varianza total y se miden en escala Likert con cuatro opciones de respuesta: "Todo el tiempo", "La mayor parte del tiempo", "Algunas veces" y "Casi nunca" (donde a mayor puntaje, mayor nivel de BS).

\section{Procedimiento}

El instrumento de evaluación se aplicó de manera colectiva en los salones de clases a los estudiantes que accedieron a participar voluntariamente en este estudio, previa aprobación de las autoridades escolares. Se les informó a los estudiantes que su participación sería anónima y se les aseguró la confidencialidad con la que sería manejada la información proporcionada por ellos. El procedimiento fue no invasivo y se siguieron las recomendaciones éticas de la investigación 
en Psicología (Sociedad Mexicana de Psicología, 2002). La tasa de rechazo fue de $11.2 \%$.

\section{RESULTADOS}

\section{Patrón de consumo}

Referente a la conducta de consumo de drogas de los estudiantes participantes en el estudio, se identificó que $63.8 \%$ ha fumado alguna vez en la vida, $62.7 \%$ ha fumado en el último año y $26.2 \%$ ha fumado en el último mes; donde la edad promedio de inicio de consumo fue de 12.6 años. En cuanto al consumo de alcohol, $60 \%$ de los estudiantes ha consumido alguna vez en su vida; $13 \%$ ha bebido más de cinco copas completas en una sola ocasión en el último año, 24.1\% ha consumido una o más copas de una a dos veces en el último año, $21.5 \%$ ha tomado una o más copas una vez en el último mes y $14.3 \%$ ha bebido una o más copas de alcohol de dos a tres veces en el último mes; con una edad de inicio de 13.5 años. Por lo que se refiere al consumo de drogas ilegales, $6.8 \%$ de los estudiantes ha consumido inhalables por lo menos una vez en su vida, 3\% ha consumido en el último año y 1.9\% en el último mes. En cuanto al consumo de marihuana, 9.6\% la ha probado alguna vez en la vida, $4.7 \%$ la ha consumido en el último año y $3 \%$ en el último mes; con una edad promedio de inicio en el consumo de drogas ilegales de 14 años (datos reportados a la fecha de aplicación del estudio).

\section{Diferencias en BS por tipo de droga}

Se realizó una prueba t para identificar si existían diferencias en las conductas de consumo de drogas en cuanto a su nivel de BS.

Tabla 1. Diferencias en la búsqueda de sensaciones por consumo de droga ilegal alguna vez en la vida.

\begin{tabular}{llll} 
& Medias & T & P \\
\hline No & .09 & & \\
Sí & .30 & -1.4 & $.000^{* *}$ \\
\hline
\end{tabular}


En la Tabla 1 se observa un nivel de BS significativamente más alto en los estudiantes que han consumido alguna droga ilegal alguna vez en la vida, en contraste con los que nunca la han consumido.

Para identificar si existían diferencias en el nivel de BS de los adolescentes de acuerdo a su frecuencia de consumo de drogas, se realizó un análisis de varianza de una sola vía, y se aplicó la prueba Post Hoc Scheffé para observar cuál de los grupos marcaba las diferencias.

Tabla 2. Diferencias en búsqueda de sensaciones y frecuencia de consumo de alcohol en el último año.

\begin{tabular}{lccccccccc} 
Variables & \multicolumn{2}{l}{ Nunca } & \multicolumn{2}{c}{ Menos 1 vez } & 1-2 veces & 3-11veces & Sig \\
\hline & M & D.S. & M & D.S. & M & D.S. & M & D.S. & \\
\hline $\begin{array}{l}\text { Búsqueda } \\
\text { sensaciones }\end{array}$ & 1.8 & .59 & 2 & .62 & 2.3 & .69 & 2.3 & .64 & $.001^{* *}$ \\
\hline
\end{tabular}

Los resultados mostraron diferencias estadísticamente significativas en la escala de BS de acuerdo a la frecuencia de consumo de alcohol en el último año. Los resultados de la prueba de Scheffé indicaron que el grupo que marca la diferencia en la BS ( $F=14.16 p<0.001)$ es el de los adolescentes que no han consumido en el último año, y que obtuvieron puntajes más bajos en su nivel de BS respecto a los que sí han consumido (Tabla 2).

Tabla 3. Diferencias en la búsqueda de sensaciones por consumo de droga ilegal.

\begin{tabular}{cccccccccc} 
Variable & \multicolumn{2}{c}{ Nunca } & \multicolumn{2}{c}{ Una vez } & \multicolumn{2}{c}{ Último año } & Último mes & Sig. \\
\hline & M & D.S. & M & D.S. & M & D.S. & M & D.S. & \\
\hline Cocaína & 1.98 & .59 & 2.5 & .67 & 2.5 & .80 & 2.7 & .65 & $.001^{* *}$ \\
\hline Inhalables & 1.98 & .66 & 2.25 & .49 & 2.6 & .75 & 2.7 & .60 & $.001^{* *}$ \\
\hline Marihuana & 1.95 & .64 & 2.32 & .74 & 2.32 & .57 & 2.65 & .66 & $.001^{* *}$ \\
\hline
\end{tabular}


En cuanto al consumo de cocaína, los resultados de la prueba indicaron que el grupo que marca la diferencia en la BS ( $F=7.80 \mathrm{p}<0.001)$ es el de los adolescentes que nunca la han consumido en su vida, quienes obtuvieron puntajes más bajos en su nivel de BS, respecto a los que sí la han consumido alguna vez en su vida, en el último año y último mes.

Respecto a los inhalables, los resultados indicaron que el grupo que marca la diferencia en la BS $(\mathrm{F}=6.64 \mathrm{p}<0.001)$ es el de los adolescentes que nunca los han probado, y que obtuvieron puntajes significativamente más bajos en su nivel de BS respecto a los que sí han consumido en el último año y último mes. En cuanto a la marihuana, los resultados de la prueba indicaron que el grupo que marca la diferencia en la BS $(F=9.71 \mathrm{p}<0.001)$ es el de los adolescentes que nunca la han consumido, y que obtuvieron puntajes significativamente más bajos en su nivel de BS respecto a los que sí la han usado alguna vez en su vida, en el último año y último mes (Tabla 3).

\section{DISCUSIÓN}

Los hallazgos de este estudio muestran que la BS está relacionada con el empleo de drogas en los adolescentes participantes, toda vez que se identificó un nivel de BS significativamente más alto en los estudiantes que han consumido alguna droga ilegal alguna vez en su vida, en comparación con los que nunca lo han hecho; así como en aquéllos con una mayor frecuencia de consumo de alcohol en el último año y una mayor frecuencia de uso de cocaína, inhalables y marihuana en el último año, de manera significativa en el último mes. Estos resultados concuerdan con lo reportado en otras investigaciones (Belin et al., 2008; Desrichard y Denarié, 2005; NCR, 2011; Pokhrel et al., 2013; Schmidt et al., 2004), en el sentido que una mayor BS en adolescentes se relaciona con consumo de drogas tanto legales (alcohol) como ilegales (cocaína, inhalables y marihuana).

El campo de estudio sobre la BS y las conductas de riesgo en la adolescencia han realizado grandes progresos en integrar el conocimiento acerca del papel de la biología en todos estos procesos, y en identificar principios científicos en los cuales basar el diseño y desarrollo apropiados de intervenciones para cambiar determinantes. Los hallazgos sugieren que el nivel de autocontrol puede funcionar como una señal de alerta, permitiendo identificar a los niños y púberes en riesgo de consumir drogas en una edad posterior (Raffaelli y 
Crockett, 2003). Por ello, se identifica la pertinencia de implementar programas preventivos para niños y preadolescentes que incluyan el fortalecimiento de habilidades protectoras del consumo, con énfasis en la habilidad de autocontrol, toda vez que se ha demostrado que son eficaces en la reducción de conductas de riesgo a largo plazo en estas poblaciones (Gavin et al., 2010).

La habilidad de autocontrol definida como: "aquellas conductas que una persona emprende de manera deliberada para lograr resultados seleccionados por ella misma; la persona debe elegir las metas y poner en práctica los procedimientos para alcanzarla" (Kazdin, 1996: 265), se ha reportado como una habilidad protectora de consumo ante un elevado nivel de BS (Cáceres et al., 2006; Pokhrel et al., 2010; Romer et al., 2010; Sussman et al., 2003); además de permitir a los adolescentes ejercer control sobre sus impulsos, inhibir su toma de riesgos, postergar gratificaciones inmediatas, planificar metas a largo plazo y ser un predictor longitudinal significativo de si los púberes se van a ver involucrados en el consumo de drogas en la adolescencia (Rafaelli y Crockett, 2003). El autocontrol es evidente a pesar de la maduración incompleta de la corteza prefrontal, factor que ha sido sugerido como una importante causa de la bs en la adolescencia (Casey et al., 2008; Steinberg, 2008).

En contraparte, la falta de autocontrol en adolescentes se ha identificado como un fuerte predictor de consumo excesivo de alcohol, tabaco y otras drogas, el mantenimiento del comportamiento de consumo, la toma de riesgos y un elevado nivel de BS (Benda, 2005; Crockett et al., 2006; López Torrecillas et al., 2003; Pérez de la Barrera, en prensa; Raffaelli y Crockett, 2003).

Es por todo lo anterior, que para retrasar el inicio y prevenir el consumo de drogas en la adolescencia, los programas preventivos deben incluir un entrenamiento exhaustivo en la habilidad de autocontrol, sin obviar un contenido informativo claro y detallado sobre drogas y sus efectos y consecuencias a corto y largo plazo (Kirby, 2008; Pérez de la Barrera, 2012). Asimismo, resulta evidente la necesidad de entrenar a los niños en esta habilidad antes de que tenga lugar el desarrollo pronunciado del sistema límbico durante la pubertad versus el de la corteza prefrontal (NCR, 2011). Por ello, estas intervenciones deben ser implementadas en la escuela secundaria. El entrenamiento en autocontrol debe practicarse de manera sistemática y de preferencia involucrar a los padres, ya que éste se inicia a través del monitoreo y refuerzo parental desde los primeros años de vida (Strayhorn, 2002). 
Respecto a las limitaciones de este estudio, la escala utilizada demostró tener niveles aceptables de confiabilidad y mostró su validez al medir la BS general en población adolescente mexicana. Sin embargo, para futuras investigaciones, cuyo objetivo sea examinar conductas de consumo de drogas, se recomienda construir una nueva escala que mida específicamente la BS hacia el consumo de drogas para adolescentes, así como una muestra más amplia y aleatoria que permita una mejor exploración del patrón de consumo de drogas en estudiantes púberes, ya que se ha comprobado que la BS alcanza su máximo nivel en la pubertad.

Por otra parte, el diseño de este estudio es de tipo transversal, lo cual limita la posibilidad de hacer conclusiones sobre inferencias causales. Por ello, se sugiere llevar a cabo estudios longitudinales, de preferencia con inicio en la infancia hasta la adolescencia, para poder identificar tendencias y evaluar de manera más confiada los efectos de la BS en la postergación de gratificaciones y, a su vez, en la toma de riesgos de los participantes.

Investigaciones futuras deberían ser dirigidas a identificar las actividades de aprendizaje que puedan ayudar a los jóvenes con altos niveles de BS a incrementar su control sobre sus impulsos antes de que experimenten consecuencias adversas. El hallazgo acerca de que los jóvenes con más memoria pueden posponer gratificaciones más fácilmente implementadas (Shamosh et al., 2008), sugiere el desarrollo de intervenciones diseñadas para incrementar el funcionamiento ejecutivo como una estrategia prometedora. Asimismo, aun cuando la BS es un rasgo típico de los adolescentes y los puede conducir a la toma de riesgo, también es fuente de otras opciones y conductas positivas. Por ello, se recomienda facilitar el involucramiento de púberes y adolescentes en otras actividades que también estimulen el sistema dopaminérgico, tales como la práctica de deportes y actividad física (Romer y Hennessy, 2007).

Finalmente, se anima a los investigadores a medir otras variables reportadas en la literatura relacionadas con la prevención de conductas de consumo de drogas en adolescentes, tales como las habilidades sociales y emocionales. Este estudio dio cuenta de la relación entre algunos comportamientos de consumo de drogas en la adolescencia y su relación con la BS; sin embargo, es evidente la necesidad de realizar más investigaciones sobre este tópico. 


\section{REFERENCIAS}

Allahverdipour, H., MacIntyre, R., Hidarnia, A., Shafii, F., Kzamnegad, A., y Ghaleiha, A. et al. (2007). Assessing protective factors against drug abuse among high school students: Self-control and the extended parallel process model. Journal of Addictions Nursing, 18, 65-73. DOI: 10.1080/10884600701334820.

Belin, D., Mar, A. C., Dalley, J. W., Robbins, T. W. y Everitt, B. J. (2008). High impulsivity predicts the switch to compulsive cocaine-taking. Science, 320, 1352-1355.

Benda, B. (2005). The robustness of self-control in relation to form of delinquency. Youth E Society, 36, 418-444. DOI: 10.1177/0044118X04268071.

Cáceres, D., Salazar, D., Tavera, I., y Tovar, J. (2006). Consumo de drogas en jóvenes universitarios y su relación de riesgo y protección con los factores de riesgo psicosociales. Universidad Psicológica de Bogotá, 5(3), 521-534.

Casey, B. J., Getz, S., y Galvan, A. (2008). The adolescent brain. Developmental Review, 28, 62-77. DOI: 10.1016/j.dr.2007.08.003.

Contreras, S.S. (2006). Factores asociados a la conducta antisocial de los menores de edad. Tesis de Doctorado no publicada. Universidad Nacional Autónoma de México.

Crockett, L., Raffaelli, M. y Ling, Y. (2006). Linking self regulation and risk proneness to risky sexual behavior: Pathways to peer pressure and early substance abuse. Faculty Publications, Department of Psychology. Paper 132.

Dahl, R. (2008). Biological, developmental and neurobehavioral factors relevant to adolescents driving risks. American Journal of Preventive Medicine, 35(3), 278-284. DOI:10.1016/j.amepre.2008.06.013.

Desrichard, O. y Denarié, V. (2005). Sensation-seeking and negative affectivity as predictors of risky behaviors: A distinction between occasional versus frequent risk-taking. Addictive Behaviors, 30, 1449-1453. DOI: 10.1016/j.addbeh.2005.01.011.

Encuesta Nacional de Adicciones 2011. (2011). Instituto Nacional de Psiquiatría Ramón de la Fuente Muñiz [INPRFM] / Secretaría de Salud. México.

Fuhrel, A., y Kalichman, S. (2006). Biological, social and psychological risk factors for HIV infection. En M. P. Bermúdez y Buela-Casal (Eds.), Recent Advances in HIV Infection Research (pp. 67-108). New York: Nova Sciences Publishers. 
Gavin, L., Catalano, R., Ferdon, C., Gloppen, K. y Markham, C. (2010). A review of positive youth development programs that promote adolescent sexual and reproductive health. Journal of Adolescent Health, 46, 75-91. DOI: 10.1016/j.jadohealth.2009.11.215.

Gervilla E. y Palmer, A. (2009). Predicción del consumo de cocaína en adolescentes mediante árboles de decisión. Revista de Investigación en Educación, 6, 7-13.

Jackson, C., Henderson, M., Frank, J. y Haw, S. (2012). An overview of prevention of multiple risk behaviour in adolescence and young adulthood. Journal of Public Health, 34, 131-140. DOI:10.1093/pubmed/ fdrl13.

Kazdin, A. (1996). Técnicas de autocontrol. Modificación de la conducta y sus aplicaciones prácticas. México: Manual Moderno.

Kirby, D. (2008). The Impact of Abstinence and Comprehensive Sex and STD/HIV Education Programs on Adolescent Sexual Behavior. Sexuality Research and Social Policy, 5(3), 6-17.

López-Torrecillas, F., Peralta, I., Muñoz-Rivas, J., y Godoy, F. (2003). Autocontrol y consumo de drogas. Revista Adicciones, 15(2), 127-136.

Martin, C., Kelly, T., Rayens, M., Brogli, B., Brenzel, A., Smith, W., et al. (2002). Sensation seeking, puberty, and nicotine, alcohol, and marijuana use in adolescence. Journal of the American Academy of Child E Adolescent Psychiatry, 41, 1495-1503.

National Research Council (2011). Looking to the Future. The Science of Adolescent Risk-Taking: Workshop Report. Washington, DC: The National Academies Press.

Palacios, J. y Cañas, J. L. (2010). Características psicosociales asociadas al consumo de alcohol, tabaco y drogas en adolescentes de Chiapas. Psicología Iberoamericana, 18(2), 27-36.

Pérez de la Barrera, C. (en prensa). Búsqueda de sensaciones y conductas sexuales de riesgo en adolescentes. Estudiantes mexicanos. Anuario de Psicología Clínica y de la Salud.

Pérez de la Barrera, C. (2012). Habilidades para la vida y consumo de drogas en adolescentes escolarizados mexicanos. Adicciones, 24(2), 153-160.

Pokhrel, P., Sussman, S., Sun, P., Kniazer, V., y Masagutov, R. (2010). Social self-control, sensation seeking and substance use in samples of US and Russian adolescents. American Journal of Health Behavior, 34(3), 374-384. 
Raffaelli, M. y Crockett, L. (2003). Sexual risk taking in adolescence: The role of self-regulation and attraction to risk. Faculty Publications, Department of Psychology. Paper 115.

Romer, D., Duckworth, A., Sznitman, Sh., y Park, S. (2010). Can adolescents learn self-control? Delay of gratification in the development of control over risk taking. Prevention Science. DOI: 10.1007/s11121010-0171-8.

Romer, D., y Hennessy, M. (2007). A biosocial-affect model of adolescent sensation seeking: The role of affect evaluation and peergroup influence in adolescent drug use. Prevention Science, 8, 89-101.

Sales, J., Smearman, E., Brody, G., Milhausen, R., Philibert, R., y Diclemente, R. (2013). Factors associated with sexual arousal, sexual sensation seeking and sexual satisfaction among female African American adolescents. Sexual Health, 10(6), 512-521.

Schmidt, V., Molina, F., Messoulam, N., y Abal, F. (2004). Búsqueda de sensaciones y su relación con el consumo de alcohol en adolescentes de nuestra población. Actas de las XI Jornadas de Investigación de la Facultad de Psicología, UBA. Buenos Aires, Argentina.

Shamosh, N. A., DeYoung, C. G., Green, A. E., Reis, D. L., Johnson, M. R., Conway, A. R. A., et al. (2008). Individual differences in delay discounting: Relation to intelligence, working memory, and anterior prefrontal cortex. Psychological Science, 19, 904-911.

Sociedad Mexicana de Psicología (2002). Código ético del psicólogo. México: Trillas.

Steinberg, L. (2008). A social neuroscience perspective on adolescent risk taking. Developmental Review, 28, 78-106.

Strayhorn, J. (2002). Self-control: Theory and research. Journal of the American Academy of Child and Adolescent Psychiatry 41(1), 7-16. DOI:http://dx.doi.org/10.1097/00004583-200201000-00006.

Sussman, S., Sun, P., McCuller, W., y Dent, C. (2003). Projects towards no drug abuse: Two-year outcomes of a trial that compares health educator delivery to selfinstruction. Preventive Medicine, 37, 155-62. DOI : 10.1016/S0306-4603(02)00222-8.

Terzian, M., Andrews, K., y Moore, K. (2011). Preventing multiple risky behaviors among adolescents: Seven strategies. Research-to-Results Brief. Tomado de Child Trendswebsite: http://www.childtrends.org/ Files/Child_Trends2011_10_01_RB_RiskyBeha viors.pdf. 
Zuckerman, M. y Neeb, M. (1980). Demographic influences in sensation seeking and expressions of sensations seeking in religion, smoking and driving habits. Personality and Individual Differences, 1, 197-206.

Zuckerman, M. (1984). Sensation seeking: A comparative approach to a human trait. Behavioral and Brain Sciences, 7, 413-471.

Zuckerman, M. (1994). Biological expressions and biosocial bases of sensation seeking. New York: Cambridge University Press.

Zuckerman, M. (2007). Sensation seeking and risky behavior. Washington: American Psychological Association.

(3) 